\title{
Molecular Clouds in the LMC Observed with NANTEN: III. Comparison with HII Regions
}

\author{
R. Abe, A. Hara, T. Hayakawa, S. Kato, A. Kawamura, A. Mizuno, N. \\ Mizuno, H. Ogawa, T. Onishi, H. Saito, K. Tachihara, K. C. Xiao, \\ N. Yamaguchi, R. Yamaguchi, and Y. Fukui \\ Department of Astrophysics, Nagoya University, Chikusa-ku, Nagoya \\ 464-8602, Japan
}

\section{Y. Yonekura}

Earth and Life Sciences, Osaka Prefecture University, Gakuencho 1-1, Sakai, Osaka 599-8531, Japan

Abstract. We have made ${ }^{12} \mathrm{CO}(J=1-0)$ observations in the LMC with NANTEN, and compared the detected giant molecular clouds (GMCs) with HII regions and stellar clusters. It is found that $\sim 80 \%$ of the GMCs are associated with HII regions. The results of comparisons of the GMCs with the HII regions and the stellar clusters are presented.

\section{Introduction}

A comparison of molecular clouds with HII regions is important for studying the process of massive-star and stellar-cluster formation. The LMC is the best target to carry out such a study in external galaxies because it is the nearest galaxy.

In the LMC, a few hundred HII regions are catalogued by Davies et al. (1976, DEM catalog) and Kennicutt \& Hodge (1986, KH catalog). The diameters range from $\sim 10 \mathrm{pc}$ to $\sim 400 \mathrm{pc}$ and the luminosities from $\sim 10^{36} \mathrm{erg} \mathrm{s}^{-1}$ to $\sim 10^{40}$ $\mathrm{erg} \mathrm{s}^{-1}$. We used these two catalogs and the stellar clusters younger than $3 \times 10^{7}$ yr (Bica et al. 1996) to compare with the GMCs.

We have made a survey for molecular clouds in the ${ }^{12} \mathrm{CO}(J=1-0)$ in the LMC, using the NANTEN 4-meter millimeter wave telescope at the Las Campanas Observatory in Chile. About 100 GMCs are newly detected, and the physical parameters are determined for 55 of them. The general properties of the GMCs are reported by Fukui et al. (1999) and Mizuno et al. (1999) in these proceedings. In this contribution, we will present the results of the detailed comparisons of the GMCs with the HII regions and the stellar clusters.

\section{Results and Discussion}

1. Association between the GMCs and the HII regions is studied. The main results are as follows; $\sim 80 \%$ of the GMCs $(43 / 55)$ are associated with HII regions. $\sim 50 \%$ of the GMCs $(26 / 55)$ are associated both with HII regions 


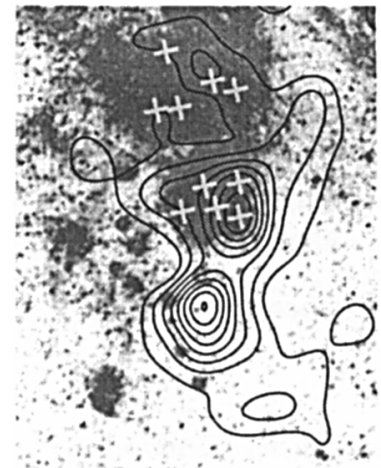

(a)

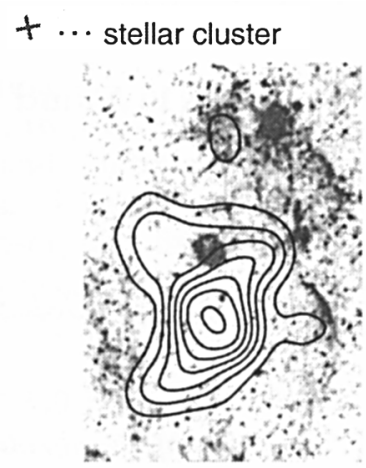

(b)

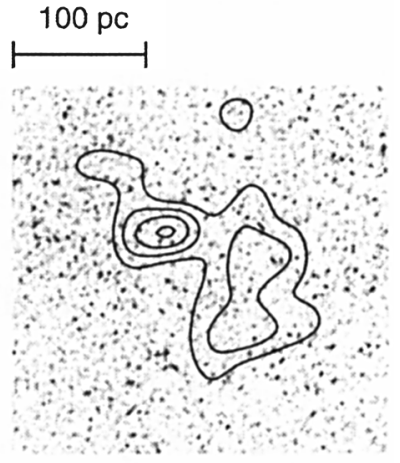

(c)

Figure 1. GMCs associated with (a) HII regions and stellar clusters (b) only HII regions (c) neither HII region nor stellar cluster.

and with stellar clusters (see Figure 1a), 30\% (17/55) only with HII regions (Figure $1 \mathrm{~b}), \sim 20 \%(12 / 55)$ neither with an HII region nor with a stellar cluster (Figure 1c). There is no GMC associated only with a stellar cluster.

2. We studied the difference in the physical parameters, such as mass and line width etc., between the GMCs that are associated HII regions and/or stellar clusters and those that are not. It is found there is no significant difference in the physical parameters between the GMCs with HII regions and/or stellar clusters and those without star formation. This indicates the GMCs associated neither with HII regions nor stellar clusters have a possibility of forming massive stars or stellar clusters in the future.

3. HII regions associated with GMCs are studied. It is found the HII regions associated with stellar clusters are larger and more luminous than those not associated with clusters.

Acknowledgments. We greatly appreciate the hospitality of all the staff members of the Las Campanas Observatory of the Carnegie Institution of Washington.

\section{References}

Bica, E., Claria, J. J., Dottori, H, Santos, J. F. C. Jr., \& Piatti, A. E. 1996, ApJS., 102, 57

Davies, R. D., Elliott, K. H., \& Meaburn, J. 1976, MmRAS, 81,89

Fukui, Y. et al. 1999, this volume

Kennicutt, R. C., \& Hodge, P. W. 1986, ApJ, 306, 130

Mizuno, N., et al. 1999, this volume 